\title{
QUALIDADE DE VIDA DOS PACIENTES COM CÂNCER ORAL: REVISÃO INTEGRATIVA DA LITERATURA
}

QUALITY OF LIFE OF PATIENTS WITH ORAL CANCER: INTEGRATIVE LITERATURE REVIEW

CALIDAD DE VIDA DE LOS PACIENTES CON CÁNCER ORAL: REVISIÓN INTEGRADORA DE LA LITERATURA

Denis Francisco Gonçalves de Oliveira ${ }^{1}$

Débora Rejane Alves Cavalcante ${ }^{2}$

Sthefane Gomes Feitosa ${ }^{3}$

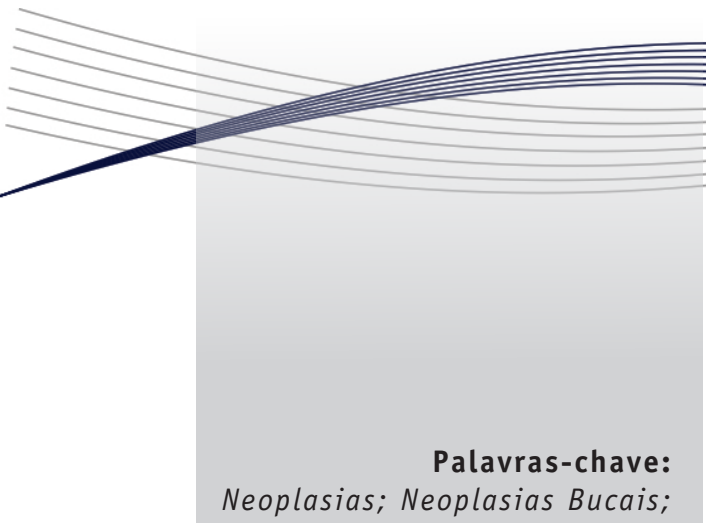

Neoplasias; Neoplasias Bucais; Qualidade de Vida.

Keywords:

Neoplasms; Mouth Neoplasms; Quality of Life.

Palabras clave: Neoplasias; Neoplasias de la Boca; Calidad de Vida.

Submetido: $12 / 06 / 2019$

Aprovado: 24/04/2020

Autor(a) para Correspondência: Denis Francisco Gonçalves de Oliveira,

R. Monsenhor Furtado, $S / N$ Rodolfo Teófilo - Fortaleza (CE)

CEP: 60441-750

E-mail:denisfgo@hotmail.com

\section{RESUMO}

o câncer oral geralmente exige tratamento cirúrgico associado ou não à radioterapia e/ou à quimioterapia, acarretando comprometimentos funcionais e estéticos que afetam a qualidade de vida dos pacientes. Portanto, neste estudo, buscou-se analisar as principais evidências sobre a qualidade de vida existentes na literatura científica e apontar quais as principais dimensões da qualidade de vida afetadas em pacientes com câncer de cavidade oral, avaliados pelo Questionário da Universidade de Washington. Para tanto, fezse uma revisão integrativa da literatura, com as seguintes etapas: adoção da questão norteadora, busca de artigos nas bases Literatura Latino-Americana e do Caribe em Ciências da Saúde (LILACS) e Medical Literature Analysis and Retrieval System Online (MEDLINE), coleta de dados, análise crítica, discussão dos resultados e construção da revisão. Foram selecionados $e$ analisados 16 artigos, sendo que os principais resultados mostraram que: a maioria dos pacientes é do gênero masculino, encontra-se no fim da $6^{a}$ década de vida, foi diagnosticada em estágios avançados, tendo mastigação, paladar, deglutição e saliva como principais domínios da qualidade de vida comprometidos. Conclui-se que é necessário considerar tais domínios afetados no planejamento terapêtico desses pacientes, para que os cuidados sejam personalizados e adequados.

1. Enfermeiro. Mestre em Ciências da Saúde. Departamento de Patologia e Medicina Legal da Universidade Federal do Ceará.E-mail: denisfgo@hotmail.com ORCID: https://orcid.org/0000-0003-0412-7300

2. Graduada em Gestão da Qualidade. Especialista em Abordagem Multidisciplinar em Oncologia pela Universidade Federal do Ceará. E-mail: deborarac@hotmail.com ORCID: https://orcid.org/0000-0002-9659-408X

3. Graduada em Odontologia. Doutoranda em Clínica Odontológica pela Universiade Federal do Ceará. E-mail: sthefane_feitosa@yahoo.com.br 0RCID: https://orcid.org/0000-0002-4254-7210 


\section{ABSTRACT}

Oral cancer usually requires surgical treatment, associated or not with radiotherapy and/or chemotherapy, leading to functional and aesthetic issues that affect patients' quality of life. Therefore, in this study, we sought to analyze the main evidence on quality of life in the scientific literature and to point out which are the main dimensions of quality of life affected in oral cavity cancer patients, assessed through the University of Washington's Questionnaire. To do this, an integrative literature review was carried out by the following steps: adoption of the guiding question, search for articles in the databases Latin American and Caribbean Literature in Health Sciences (LILACS) and Medical Literature Analysis and Retrieval System Online (MEDLINE), data collection, critical analysis, discussion of results, and building of the review. Sixteen articles were selected and analyzed, and the main results have showed that: most patients are male, at the end of the 6th decade of life, diagnosed in advanced stages, and they have chewing, taste, swallowing, and saliva as the main quality of life domains affected. It is concluded that there is a need to consider such affected domains in the therapeutic planning for these patients, so that their care is individualized and adequate.

\section{RESUMEN}

El cáncer oral generalmente requiere tratamiento quirúrgico, asociado o no con radioterapia y/o quimioterapia, lo que causa problemas funcionales y estéticos que afectan la calidad de vida de los pacientes. Por lo tanto, en este estudio, se busca analizar la evidencia principal acerca de la calidad de vida en la literatura científica y señalar cuáles son las principales dimensiones de la calidad de vida afectadas en pacientes con cáncer de cavidad oral, evaluados por el Cuestionario de la Universidad de Washington. Con este fin, se realizó una revisión integradora de la literatura, con los siguientes pasos: adopción de la pregunta guía, búsqueda de artículos en las bases Literatura Latinoamericana y del Caribe en Ciencias de la Salud (LILACS) y Medical Literature Analysis and Retrieval System Online (MEDLINE), recopilación de datos, análisis crítico, discusión de resultados y construcción de la revisión. Se seleccionaron y analizaron 16 artículos y los principales resultados mostraron que: la mayoría de los pacientes son hombres, al final de la $6^{a}$ década de vida, diagnosticada en etapas avanzadas, con masticación, gusto, deglución y saliva como los principales dominios de la calidad de vida afectados. Se concluye que es necesario considerar dichos dominios afectados en la planificación terapéutica de estos pacientes, para que la atención sea personalizada y adecuada.

\section{INTRODUÇÃO}

No contexto mundial, o câncer se destaca entre as principais doenças no cenário atual. No quesito mortalidade, ocupa a $2^{\mathrm{a}}$ posição no ranking universal, com incidência global em 2018 de pelo menos 18,1 milhões de novos casos, responsável por 9,6 milhões de mortes $^{1-2}$. Quanto às principais localizações de acometimento, o câncer de mama é o mais comum no sexo feminino; já no masculino, o mais prevalente é o de pulmão, seguido pelo da próstata².

Em relação ao câncer de cavidade oral, consultando os dados disponíveis mais atuais, as estimativas são de que no mundo, em 2018, pelo menos 354.864 pessoas tenham tido a doença, que teria causado 177.384 óbitos, figurando entre os 20 principais tipos de câncer no cenário global².

No Brasil, o número de casos novos de câncer oral para cada ano do triênio 2020-2022 será de
11.180 em homens e de 4.010 em mulheres, com o país ocupando a $5^{a}$ posição na população masculina e a $13^{a}$ na feminina, o que corresponde a um risco de 10,69 casos novos a cada 100 mil homens e 3,71 casos a cada 100 mil mulheres ${ }^{3}$.

As localizações afetadas são: lábios, gengivas, mucosa jugal, palato, língua, assoalho da boca, glândulas salivares e orofaringe. No entanto, não há consenso sobre os locais que compõem a definição de câncer oral, sendo língua e assoalho da boca os mais comumente afetados. Vale ressaltar que a distribuição da localização varia de acordo com os fatores de risco associados ${ }^{3-4}$, sendo os principais o tabagismo e o consumo excessivo de álcool, que têm efeitos sinérgicos. Além desses, destaca-se, também, a exposição solar, associada principalmente com câncer de lábio, infecção pelo papiloma vírus humano (HPV), comumente combinada ao câncer de orofaringe, e fatores ocupacionais ${ }^{3,5}$. Quanto ao tipo 
histológico, o carcinoma de células escamosas é o mais comum, correspondendo a cerca de $90 \%$ das lesões orais malignas ${ }^{4}$.

Clinicamente, o câncer de boca pode manifestarse como úlcera com bordas endurecidas que não cicatriza por mais de 15 dias, leucoplasia, eritroplasia, eritroleucoplasia, massa exofítica e/ou lesão endofítica. 0 estadiamento clínico se baseia no tamanho do tumor, no envolvimento de linfonodos e no desenvolvimento de metástase, sendo adotado o termo tumor-nódulo-metástase (TNM). No momento do diagnóstico, muitos pacientes são classificados em estágios avançados devido ao fato da doença ser geralmente assintomática nos estágios iniciais, o que leva à procura tardia por atendimento e, muitas vezes, isso compromete o prognóstico. Além disso, nos estágios avançados, o paciente pode apresentar sangramento oral, perda de dentes, disfagia e odinofagia ${ }^{5-6}$.

Sobre o tratamento, a Portaria n. 516, de 17 de junho de 2015, da Secretaria de Atenção à Saúde do Ministério da Saúde (SAS/MS), aprovou as diretrizes diagnósticas e terapêuticas do câncer de cabeça e pescoço. Em relação ao tratamento do carcinoma de células escamosas de cavidade oral em estágios iniciais, a orientação é a ressecção cirúrgica da região acometida, com margem de segurança de $1 \mathrm{~cm}$. Quanto aos casos de carcinoma de células escamosas de orofaringe em estágios iniciais, a recomendação é cirurgia ou radioterapia, sendo necessário considerar em cada caso as prováveis sequelas do tratamento. Já em casos de câncer de cavidade oral em estágios avançados, a orientação é cirurgia, seguida ou não por radioterapia adjuvante, ao passo que a recomendação para o tratamento de câncer de orofaringe em estágios avançados é a irradiação isolada ou a radioquimioterapia ${ }^{7}$. Destaca-se que, em geral, o tratamento do câncer de cavidade oral provoca sequelas funcionais $e$ estéticas, sendo particularmente incapacitante em relação à aparência, à função oral e à mobilidade do ombro, levando os pacientes e suas famílias ${ }^{8}$ ao sofrimento psíquico.

Por tais razões, avaliar a qualidade de vida de pacientes com câncer oral se mostra importante para observar os impactos da doença e do tratamento na vida do paciente. Ressalta-se que, para a Organização Mundial da Saúde (OMS), a qualidade de vida não se limita à ausência de doença ou enfermidade, mas engloba a capacidade de um indivíduo viver de forma produtiva e agradável, sendo, portanto, um conceito

\section{Clinicamente, o câncer de boca pode manifestar-se como úlcera com bordas endurecidas que não cicatriza por mais de 15 dias...}

subjetivo e multidimensional que inclui os domínios da saúde física, mental e social, a satisfação com o tratamento, as preocupações sobre o futuro e o bemestar geral. Para avaliá-la há vários questionários, dentre os quais a versão 4 do Questionário da Universidade de Washington [University of Washington Quality of Life Questionnaire (UWQOL) $]^{9}$, um dos mais aplicados a pacientes com câncer oral, considerando seu bem-estar geral, e já traduzido para mais de 30 idiomas.

Criado em 1993, o UW-Q0L passou por 3 modificações consecutivas e a quarta foi validada para ser aplicada no Brasil. Breve e abrangente, trata-se de um dos instrumentos mais usados para avaliar a qualidade de vida de pacientes com câncer de cabeça e pescoço. Suas 12 questões visam a avaliar 12 dimensões da qualidade de vida: dor, aparência, atividade, recreação, deglutição, mastigação, fala, ombros, paladar, saliva, humor e ansiedade. Possibilita, ainda, que o paciente expresse outros pontos que achar relevantes para sua qualidade de vida. Cada questão pontua de 0 a 100: quanto maior o escore, melhor a qualidade de vida; quanto menor, mais acometimento ele apresenta nesse domínio $0^{9-10}$.

Considerando, portanto, a importância de avaliar a qualidade de vida dos pacientes com câncer oral, buscou-se neste estudo analisar as principais evidências de qualidade de vida identificadas na literatura científica e apontar quais são as principais dimensões da qualidade de vida afetadas em pacientes com câncer de cavidade oral avaliadas pelo UW-QOL.

\section{METODOLOGIA}

Trata-se de estudo descritivo de revisão integrativa da literatura. Para a realização da pesquisa foram percorridas as seguintes etapas ${ }^{11}$ : a) identificação do tema e da questão norteadora; b) busca de artigos; c) coleta de dados; d) análise crítica dos estudos; e) discussão dos resultados; e f) apresentação da revisão integrativa. 
A questão norteadora adotada foi: Quais são as principais dimensões da qualidade de vida afetadas em pacientes com câncer de cavidade oral?

0 levantamento dos artigos científicos foi feito na base de dados Literatura Latino-Americana e do Caribe em Ciências da Saúde (LILACS) e no Sistema Online de Busca e Análise de Literatura Médica (MEDLINE). Para a busca foram adotados os descritores "qualidade de vida" e "neoplasias bucais", tanto em português quanto em inglês, sendo também empregado o operador booleano "AND". Os critérios de inclusão englobaram artigos: a) publicados em língua inglesa ou portuguesa; b) que abordassem o tema em questão; c) que adotassem o questionário UW-QOL; e d) que tivessem sido publicados nos últimos 5 anos (no período de 2015 a 2019 ). 0 critério de exclusão foi caracterizar-se como artigos de revisão de literatura. Dessa forma, o total de artigos identificados foi 1.544. Com a aplicação dos critérios de inclusão, esse número se reduziu a 44 . Excluindo os 28 artigos de revisão de literatura, chegou-se aos 16 artigos incluídos em nossa revisão integrativa.

Tal processo de seleção é resumido na Figura 1.

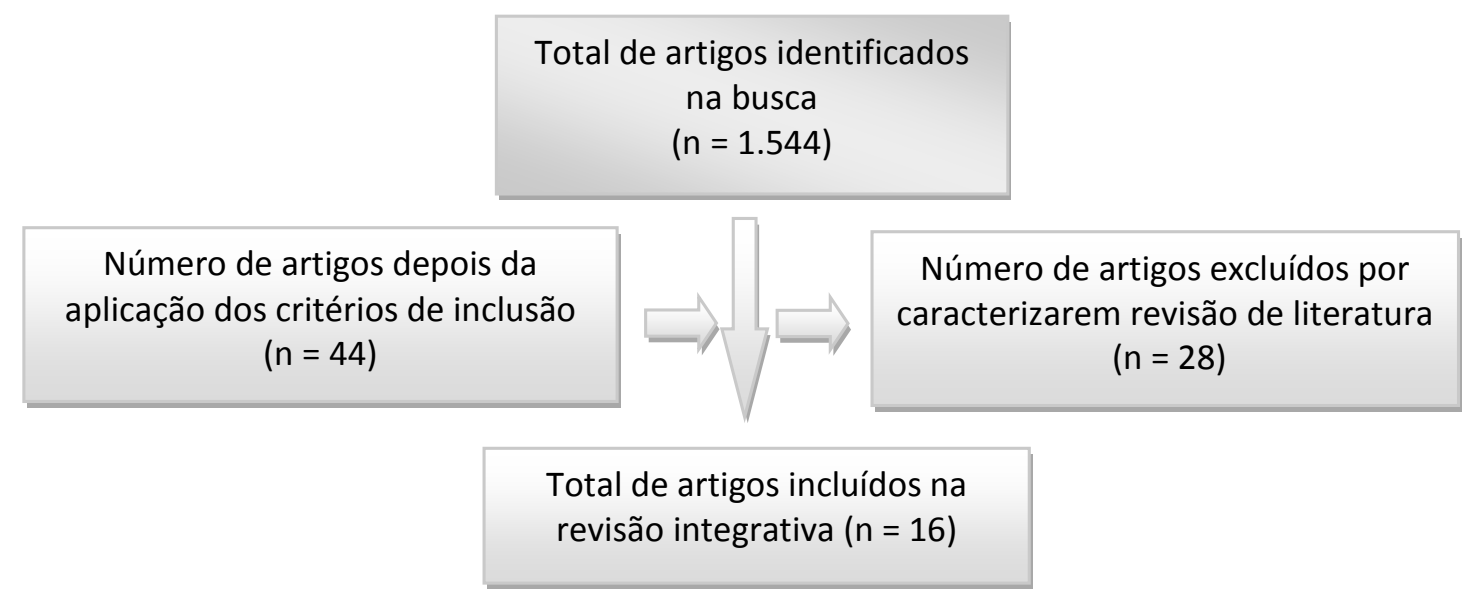

Figura 1 - Fluxo de seleção dos artigos.

Fonte: Elaborada pelos autores.

Vale ressaltar que este estudo teve início em março de 2019, com a elaboração do projeto de pesquisa, sendo as buscas e a análise dos artigos feitas em abril do mesmo ano, assim como a redação da revisão integrativa foi feita no mês seguinte. Partindo das informações obtidas foram elaborados os quadros e as figuras que ilustram os achados desta pesquisa.

\section{RESULTADOS}

Nesta revisão integrativa foram analisados 16 artigos científicos: 2 publicados em 2015; 7, em 2016; 3, em 2017; e 4, em 2018 (Quadro 1).

Quadro 1 - Relação dos artigos incluídos na revisão integrativa. Fortaleza, 2019.

\begin{tabular}{|l|l|l|}
\hline Titulo do artigo & Ano & Periódico \\
\hline How will I be after my operation for oral cancer? & 2015 & $\begin{array}{l}\text { British Journal of Oral and } \\
\text { Maxillofacial Surgery }\end{array}$ \\
\hline $\begin{array}{l}\text { Prospective study of the influence of psychological and medical } \\
\text { factors on quality of life and severity of symptoms among patients } \\
\text { with oral squamous cell carcinoma }\end{array}$ & 2015 & $\begin{array}{l}\text { British Journal of Oral and } \\
\text { Maxillofacial Surgery }\end{array}$ \\
\hline $\begin{array}{l}\text { Functional assessments in patients undergoing radial forearm flap } \\
\text { following hemiglossectomy }\end{array}$ & 2016 & Journal of Craniofacial Surgery \\
\hline $\begin{array}{l}\text { Reconstruction of tongue defects with the contralateral nasolabial } \\
\text { island flap }{ }^{15}\end{array}$ & 2016 & $\begin{array}{l}\text { Journal of Oral and Maxillofacial } \\
\text { Surgery }\end{array}$ \\
\hline
\end{tabular}




\begin{tabular}{|c|c|c|}
\hline Título do artigo & Ano & Periódico \\
\hline $\begin{array}{l}\text { Radial free forearm flap versus pectoralis major pedicled flap } \\
\text { for reconstruction in patients with tongue cancer: assessment of } \\
\text { quality of life }{ }^{16}\end{array}$ & 2016 & $\begin{array}{l}\text { Medicina Oral, Patología Oral y } \\
\text { Cirugía Bucal }\end{array}$ \\
\hline $\begin{array}{l}\text { Health-related quality of life after treatment for neoplasia of the } \\
\text { major salivary glands: a pilot study }{ }^{17}\end{array}$ & 2016 & $\begin{array}{l}\text { British Journal of Oral and } \\
\text { Maxillofacial Surgery }\end{array}$ \\
\hline $\begin{array}{l}\text { Outcomes by area of residence deprivation in a cohort of oral } \\
\text { cancer patients: survival, health-related quality of life, and place } \\
\text { of death }{ }^{18}\end{array}$ & 2016 & Oral Oncology \\
\hline $\begin{array}{l}\text { Factors that affect the quality of life of patients with oral cancer } \\
\text { who have had their defects reconstructed immediately after excision } \\
\text { of the tumour }\end{array}$ & 2016 & $\begin{array}{l}\text { British Journal of Oral and } \\
\text { Maxillofacial Surgery }\end{array}$ \\
\hline $\begin{array}{l}\text { Can superselective intra-arterial chemoradiotherapy replace surgery } \\
\text { followed by radiation for advanced cancer of the tongue and floor } \\
\text { of the mouth? } \text { ? }^{20}\end{array}$ & 2016 & $\begin{array}{l}\text { Journal of Oral and Maxillofacial } \\
\text { Surgery }\end{array}$ \\
\hline $\begin{array}{l}\text { Quality of life in long-term oral cancer survivors: an 8-year } \\
\text { prospective study in China }\end{array}$ & 2017 & $\begin{array}{l}\text { Oral Surgery, Oral Medicine, Oral } \\
\text { Pathology and Oral Radiology }\end{array}$ \\
\hline $\begin{array}{l}\text { Quality of life outcome measures using University of Washington } \\
\text { questionnaire version } 4 \text { in early } T 1 / T 2 \text { anterior tongue cancers with } \\
\text { and without radiotherapy: A cross-sectional study }\end{array}$ & 2017 & Indian Journal of Cancer \\
\hline $\begin{array}{l}\text { Importance of chewing, saliva, and swallowing function in } \\
\text { patients with advanced oral cancer undergoing preoperative } \\
\text { chemoradiotherapy: a prospective study of quality of life }{ }^{22}\end{array}$ & 2017 & $\begin{array}{l}\text { International Journal of Oral } \\
\text { and Maxillofacial Surgery }\end{array}$ \\
\hline $\begin{array}{l}\text { Health-related quality of life in patients with major salivary gland } \\
\text { carcinoma }\end{array}$ & 2018 & $\begin{array}{l}\text { European Archives of Oto-Rhino- } \\
\text { Laryngology }\end{array}$ \\
\hline $\begin{array}{l}\text { Assessment of quality of life in patients with advanced oral } \\
\text { cancer who underwent mandibulectomy with or without bone } \\
\text { reconstruction }{ }^{24}\end{array}$ & 2018 & $\begin{array}{l}\text { Revista da Associação Médica } \\
\text { Brasileira }\end{array}$ \\
\hline $\begin{array}{l}\text { Effect of induction chemotherapy on the quality-of-life in patients } \\
\text { with advanced stage tongue cancer by a prospective study }{ }^{25}\end{array}$ & 2018 & $\begin{array}{l}\text { Journal of International Medical } \\
\text { Research }\end{array}$ \\
\hline $\begin{array}{l}\text { Patient-reported quality of life outcomes following treatment for } \\
\text { oral cancer }{ }^{26}\end{array}$ & 2018 & $\begin{array}{l}\text { International Journal of Oral } \\
\text { and Maxillofacial Surgery }\end{array}$ \\
\hline
\end{tabular}

Fonte: Elaborado pelos autores.

Os estudos foram conduzidos em 7 países: China (6), Reino Unido (4), Alemanha (2), Índia (1), Áustria (1), Japão (1) e Brasil (1). No que tange à sua tipologia, verificou-se que 6 eram transversais; 10, longitudinais; 9, prospectivos; e 1, retrospectivo. Destaca-se que, em relação aos autores, no cômputo geral participaram 99 , levando a uma média de 6 autores por artigo científico.

Ressalta-se que, além do questionário UW-Q0L, 3 estudos usaram mais de 1 questionário e 1 estudo mais 2 questionários para avaliar a qualidade de vida de seus pacientes. Tais questionários adotados foram: Freiburg Questionnaire on Coping with Illness (FQCI); Brief Symptom Inventory (BSI); 14-Item Oral Health Impact Profile (0HIP-14); Patient Concerns Inventory (PCI); Functional Assessment of Cancer Therapy - Head \& Neck version 4 questionnaire (FACT-H\&N).

Sobre a amostra adotada nos estudos, observou-se que a média do número de sujeitos que participaram das pesquisas foi de 79 pacientes (variando nos estudos de 7 a 633). Destaca-se que 65,5\% dos incluídos nos estudos eram do sexo masculino e $34,5 \%$, do feminino, com idade média de 58,2 anos. A respeito do estágio clínico da doença, a grande maioria $(62,2 \%)$ se encontrava em estágio avançado e apenas $37,8 \%$, nos estágios iniciais da doença. No que se refere ao tratamento, $45 \%$ deles se submeteram apenas à cirurgia, $51 \%$ fizeram 
cirurgia e terapia adjuvante (radioterapia e/ou quimioterapia) e apenas $4 \%$ se submeteram a radioterapia e quimioterapia.

Sobre a qualidade de vida desses pacientes, pode-se afirmar que os menores escores foram encontrados: a) depois do tratamento, em comparação aos escores antes do tratamento; b) em pacientes que fizeram cirurgia associada à terapia adjuvante, principalmente radioterapia; c) em pacientes com idade acima de 65 anos; d) que residiam em áreas socioeconomicamente desfavorecidas; e) que viviam sozinhos; e f) que foram diagnosticados nos estágios III/IV.

Já os melhores escores de qualidade de vida foram obtidos: a) por pacientes diagnosticados nos estágios I/II; b) que não necessitaram de radioterapia; e c) que fizeram esvaziamento cervical seletivo, em vez de esvaziamento radical. Destaca-se que, quando o tratamento se associava ao esvaziamento radical, os principais domínios afetados eram a aparência e o movimento dos ombros.

Vale ressaltar que, em relação aos principais domínios avaliados pelo UW-QOL, os mais comprometidos nos pacientes com câncer de cavidade oral foram mastigação, paladar, deglutição e saliva (Figura 2), ao passo que os menos afetados foram dor, ansiedade e humor.

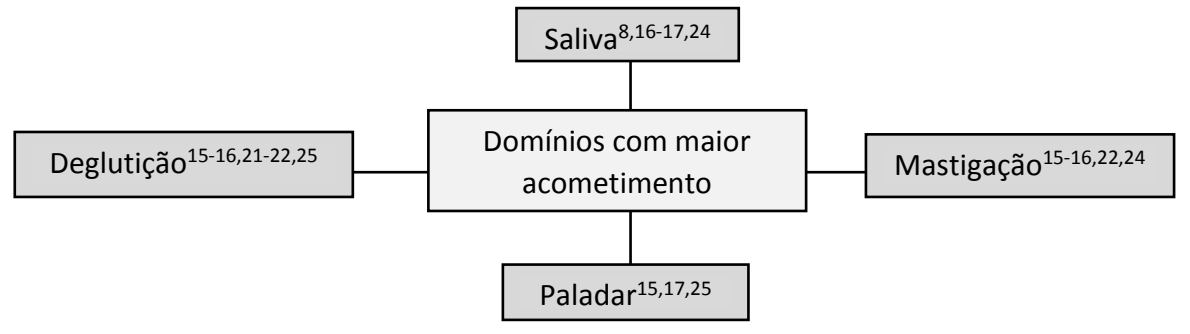

Figura 2 - Principais domínios afetados.

Fonte: Elaborada pelos autores.

\section{DISCUSSÃO}

No cenário mundial, o câncer de cavidade oral atinge principalmente a população masculina, sobretudo acima dos 40 anos. No Brasil, as estimativas para o triênio 2020-2022 são de que a doença ocupe a $5^{a}$ posição na população masculina e a $13^{a}$ na feminina, correspondendo à neoplasia maligna mais comum da região de cabeça e pescoço, adquirindo, assim, relevante importância no cenário da saúde pública ${ }^{3}$.

Nesta revisão, observou-se que a grande maioria dos pacientes que compôs a amostra nos estudos era do sexo masculino. Esse dado reflete a epidemiologia geral para o câncer de cavidade oral no contexto global e no Brasil, onde o sexo masculino é o mais acometido. Vale ressaltar que a incidência geral na população masculina foi de 246.420 casos em 2018, perfazendo quase $70 \%$ da incidência geral dessa neoplasia ${ }^{2}$. Esse maior acometimento da população masculina pode estar associado a maior exposição desse público aos fatores de risco, principalmente o tabagismo e o etilismo, podendo ter relação, ainda, com sua resistência em buscar os serviços de saúde, sobretudo quando o objetivo é criar medidas preventivas e voltadas à educação em saúde, o que pode dificultar a conscientização sobre os fatores de risco e retardar o diagnóstico do câncer oral.

Quanto à faixa etária, nesta revisão, a média se concentrou no fim da $6^{a}$ década de vida, o que corrobora o comportamento epidemiológico dessa neoplasia na população mundial, cuja faixa etária com maior incidência corresponde ao intervalo entre a $5^{\text {a }}$ e a $7^{\text {a }}$ década de vida ${ }^{5}$. Nessa faixa etária foram estimados, para o ano de $2018,228.238$ casos, correspondendo a $64 \%$ de todos os casos no mundo². Esse perfil de prevalência nas faixas etárias mais avançadas pode ser explicado pelo acúmulo de danos celulares e, consequentemente, por gerar alterações patológicas no indivíduo. Vale ressaltar que o desenvolvimento de neoplasias malignas é um processo complexo que pode demandar várias décadas. Outro ponto a considerar é que as células são expostas constantemente a vários fatores citotóxicos e mutagênicos que podem danificar o DNA. No entanto, proteínas e alguns genes, como oncogenes, genes supressores de tumor e genes de reparo de DNA, contribuem em um processo complexo para manter a homeostase celular. Alterações e inativação dessas proteínas e genes podem então favorecer o desenvolvimento de câncer ${ }^{6}$. 
A respeito do estágio clínico, percebeu-se que a maioria dos pacientes incluídos nesta revisão foi diagnosticada em estágio tardio, fato que já acarreta maior acometimento da qualidade de vida e que também exigirá tratamentos mais agressivos, que, em consequência, impactarão ainda mais a condição de vida. Esse dado também demonstra a necessidade do diagnóstico precoce nos serviços de saúde, o que deveria ocorrer devido à boca ser uma área de fácil avaliação pelos pacientes e profissionais da saúde e que deveria possibilitar o diagnóstico da doença nos estágios $\mathrm{I} / \mathrm{II}^{5}$. É importante destacar que, nos estágios iniciais, o câncer oral pode ser assintomático, o que muitas vezes colabora para um diagnóstico tardio. Aponta-se que algumas das manifestações clínicas apresentadas pelos pacientes com câncer de cavidade oral são lesões potencialmente malignas, como leucoplasia, eritroplasia, eritroleucoplasia e queilite actínica, que acometem sobretudo a borda lateral e a superfície ventral da língua, seguida pelo assoalho da boca e pelo lábio inferior ${ }^{5-6}$. Dessa forma, são necessárias condutas que visem à prevenção da doença, buscando-se evitar os fatores de risco e favorecendo o diagnóstico precoce.

Salienta-se que o tabagismo é o principal fator de risco, pois o tabaco contém mais de 50 substâncias com potencial carcinogênico. Já o álcool atua como agente potencializador. Essa associação aumenta em até 30 vezes os riscos do desenvolvimento de câncer oral. Outros fatores de risco são: o HPV, em particular o tipo 16, associado com o câncer de orofaringe; e a exposição solar, em especial das 10:00 às 16:00, estando relacionado com o câncer de lábio $0^{3-5}$. Dessa forma, são fundamentais estratégias de educação em saúde que promovam a conscientização da população sobre esses fatores de risco, para que as pessoas adotem comportamentos que evitem ou reduzam a exposição a eles.

No que diz respeito ao diagnóstico precoce, mostra-se fundamental uma detalhada anamnese, incluindo avaliação extra e intraoral adequada e minuciosa por parte dos profissionais da saúde, em especial nas localizações mais afetadas, bem como estimular o autoexame dos pacientes, com campanhas educativas e ações de rastreamento. Outra conduta importante é a realização de biópsia de lesões que persistam por mais de duas semanas, como forma de descartar ou confirmar o diagnóstico de câncer de cavidade oral ${ }^{5-6,27}$.

Em relação ao tratamento, nota-se que a grande maioria dos pacientes incluídos nesta revisão

\section{...a maioria dos pacientes incluídos nesta revisão foi diagnosticada em estágio tardio...}

integrativa se submeteu a cirurgia associada a terapia adjuvante. Nesse ponto, enfatiza-se que 0 tratamento deve ser adequado às necessidades de cada paciente: nos tumores em estágios iniciais, o tratamento consistirá principalmente em cirurgia e/ou radioterapia, sendo a quimiorradioterapia adotada em especial nos tumores em estágios clínicos avançados ou quando se objetiva a preservação de órgãos ${ }^{28}$. Cabe enfatizar que a cirurgia isolada não é mais a escolha ideal para a maioria dos cânceres orais, devido aos resultados inferiores do tratamento e tendo em vista que a grande maioria dos pacientes é diagnosticada em estágios tardios. Atualmente, a cirurgia só é oferecida para o câncer bucal em estágio inicial sem disseminação para linfonodos e sem evidência clínica ou radiográfica de metástase $e^{29}$. Dessa forma, como nesta revisão a maioria dos pacientes se encontrava nos estágios III/IV, esperase maior proporção de casos tratados com cirurgia associada a radioterapia e/ou a quimioterapia. Salienta-se que, segundo as diretrizes da SAS/MS, o tratamento para pacientes em estágios avançados envolve cirurgia seguida ou não de radioterapia em casos de câncer de cavidade oral e irradiação isolada ou radioquimioterapia em casos de câncer de orofaringe?.

A respeito dos domínios da qualidade de vida, percebeu-se que os mais afetados nos estudos incluídos na revisão foram: mastigação, paladar, deglutição e saliva. As causas para alterações nesses domínios guardam relação tanto com a lesão neoplásica quanto com o tratamento empregado. Cabe ressaltar que a cavidade oral apresenta diversas estruturas anatômicas diferentes que trabalham de forma coordenada para executar várias funções. Dessa forma, a língua tem papel na deglutição ao comprimir e pressionar os alimentos contra o palato, que leva à formação do bolo alimentar. Também atua no paladar, pois tem papilas em sua superfície dorsal, além de ser o órgão articulador mais importante da fala. As glândulas salivares contribuem produzindo saliva, líquido viscoso e mucoide que contribui para a lubrificação e a umidade da cavidade oral. 
Quanto aos dentes, eles têm papel fundamental na mastigação, pois rasgam e moem os alimentos em pedaços pequenos, contribuindo para o processo de digestão ${ }^{30}$. Dessa forma, o acometimento dessas estruturas acaba ocasionando comprometimento de domínios da qualidade de vida diretamente associados com essas estruturas anatômicas.

Salienta-se, ainda, que dor, ansiedade e humor geralmente foram os domínios menos afetados. É fato que esse perfil pode variar dependendo do estudo considerado. E o que se percebe é que tais domínios podem ter boas respostas com o manejo adequado, visto que a dor pode ser aliviada com o tratamento da neoplasia maligna e com medidas farmacológicas e não farmacológicas, e que a depressão e a ansiedade podem ter um melhor controle dependendo do apoio familiar recebido ao longo do tempo, além do apoio emocional oferecido pela equipe e da própria resiliência do paciente. No entanto, mostrase importante considerar outros pontos a respeito desses domínios. Um fato relevante evidenciado em um dos estudos é que no grupo feminino o domínio ansiedade, em geral, tem escore menor do que na população masculina, o que não pode deixar de ser considerado no planejamento terapêutico $0^{8,23}$.

Em relação às sequelas associadas ao tratamento, tanto a cirurgia quanto o tratamento adjuvante afetam a qualidade de vida dos pacientes. Destacase que a cirurgia pode afetar: a fala, que pode ter comprometimento tanto temporário, relacionado ao edema, quanto a longo prazo, por alterações estruturais; e a deglutição, devido à diminuição da parede da faringe e à redução da mobilidade da língua e da mandíbula ${ }^{31}$. Já a radioterapia apresenta sérias complicações que afetam as estruturas orais, como glândulas salivares, ossos, dentição e mucosa oral, dentre outras, com consequências clínicas para o paciente, como mucosite, xerostomia, osteorradionecrose e cárie por radiação $0^{6}$. Sobre os eventos adversos relacionados à quimioterapia, além de anemia, perda de audição, alopecia e fadiga, deve-se destacar os relativos à cavidade oral: sangramento, hipossalivação, infecções, dor e alterações do paladar ${ }^{28}$.

Assim, considerando tanto as alterações funcionais decorrentes do câncer de boca quanto do tratamento adotado, é importante conhecer as principais dimensões da qualidade de vida alteradas, como destacadas neste estudo, para 0 direcionamento das medidas terapêuticas. Para isso, é necessário o uso de questionários validados para

\section{Constatou-se que a maioria dos pacientes diagnosticados com câncer de cavidade oral é do gênero masculino...}

a avaliação de todas as dimensões da qualidade de vida dos pacientes. Tais recursos podem ser adotados tanto antes quanto durante e depois do tratamento, podendo avaliar tanto o impacto da doença quanto o comprometimento gerado pelo tratamento. Vale ressaltar que uma opção de questionário validado para o uso no contexto brasileiro é o UW-QOL, desenvolvido para pacientes com câncer de cabeça e pescoço e, portanto, pode ser aplicado a pacientes com câncer oral. Outra consideração relevante é a existência de opções de questionários, dentre eles os citados nos estudos incluídos nesta revisão integrativa. No entanto, deve-se levar em conta que tais instrumentos precisam ser validados para o contexto em que serão empregados, para que de fato possibilitem uma avaliação adequada, incluindo etapas como tradução, adaptação cultural, teste/ reteste e validade externa em estudos prévios, antes de sua inclusão na prática assistencial?

\section{CONCLUSÃO}

Constatou-se que a maioria dos pacientes diagnosticados com câncer de cavidade oral é do gênero masculino, principalmente no fim da $6^{\text {a }}$ década de vida e em estágios avançados, o que exige tratamentos mais agressivos, como cirurgia associada a radioterapia e/ou quimioterapia.

Os principais domínios da qualidade de vida afetados em tais pacientes foram mastigação, paladar, deglutição e saliva, uma vez que tais funções estão relacionadas diretamente com estruturas fundamentais da cavidade oral, tendo comprometimento causado tanto pela neoplasia maligna quanto pelo tratamento selecionado, seja cirurgia, radioterapia ou quimioterapia.

Assim, mostra-se necessária a adoção de cuidados com tais domínios da qualidade de vida, de forma a reduzir os impactos e melhorar a adaptação do paciente à nova condição de vida. Deve-se considerar também a inclusão dos questionários de qualidade de vida na prática assistencial, visto ser necessário avaliar os 
domínios afetados em cada paciente, possibilitando uma assistência individualizada e centralizada nele. Para tanto, deve-se adotar questionários validados para cada cenário específico. Em nosso caso, que sejam adequados para a realidade brasileira. E dentre os vários questionários se destaca o UW-Q0L, já validado para ser usado em nosso contexto, além de ser prático para acompanhar os pacientes com câncer oral. Outro ponto importante é o apoio da equipe de saúde e dos familiares, que faz toda a diferença em alguns domínios da qualidade de vida, como no da ansiedade e no da depressão.

Também é relevante pensar em medidas preventivas que possam contribuir tanto para a redução da exposição do paciente aos fatores de risco quanto para o diagnóstico precoce das lesões. Para isso, além de ações educativas voltadas à conscientização da comunidade a respeito dos fatores de risco e ao autoexame, a capacitação dos profissionais da saúde é essencial para que possam identificar a doença em seus estágios iniciais, possibilitando um tratamento em tempo hábil e menos agressivo, com menor comprometimento da qualidade de vida desses pacientes.

\section{CONTRIBUIÇÃO DOS AUTORES}

Denis Francisco Gonçalves de Oliveira contribuiu com a realização da pesquisa, o delineamento do estudo e a redação do manuscrito. Débora Rejane Alves Cavalcante contribuiu com a realização da pesquisa e o delineamento do estudo. Sthefane Gomes Feitosa contribuiu com a realização da pesquisa, o delineamento do estudo e a redação e revisão crítica do manuscrito.

\section{REFERÊNCIAS}

1. Organização Pan-Americana da Saúde. Folha informativa: câncer [document on the internet]. 2018 [cited 2020 May 14]. Available from: https:// www.paho.org/bra/index.php?option $=$ com con tent \&view $=$ article \&id $=5588:$ folha-informativa cancer\&Itemid $=1094$

2. Ferlay J, Colombet M, Soerjomataram I, Mathers C, Parkin DM, Piñeros M, et al. Estimating the global cancer incidence and mortality in 2018: GLOBOCAN sources and methods. Int J Cancer. $2019 ; 144(8)$ :194153.

3. Instituto Nacional de Câncer. Estimativa 2020: incidência de câncer no Brasil. Rio de Janeiro: INCA; 2019.
4. El-Naggar AK, Chan JKC, Grandis JR, Takata T, Slootweg PJ. WHO Classification of Head and Neck Tumours. 4. ed. Lyon: IARC Press; 2017.

5. Domingos PAS, Passalacqua MLC, Oliveira ALBM. Câncer bucal: um problema de saúde pública. Rev Odontol Univ Cid São Paulo [serial on the internet]. 2014 [cited 2020 May 14];26(1):46-52. Available from: http://arquivos.cruzeirodosuleducacional. edu.br/principal/old/revista odontologia/pdf/ janeiro abril_2014/0donto $26 \quad 2014 \quad 46$ 52.pdf

6. Mateo-Sidron Anton MC, Somacarrera Perez ML. Cáncer oral: genética, prevención, diagnóstico y tratamiento. revisión de la literatura. Av 0dontoestomatol. 2015;31(4):247-59.

7. Brasil. Portaria n. 516 [document on the internet]. 2015 Jun 17 [cited 2020 Mar 26]. Available from: http://bvsms.saude.gov.br/bvs/saudelegis/ sas/2015/prt0516 $1706 \quad 2015 . h t m l$

8. Yan YB, Meng L, Liu ZQ, Xu JB, Liu H, Shen J, et al. Quality of life in long-term oral cancer survivors: an 8-year prospective study in China. Oral Surgery, Oral Medicine, Oral Pathology and Oral Radiology. 2017;123(1):67-75.

9. Vartanian JG, Carvalho $A L$, Yueh $B$, Furia $C L$, Toyota J McDowell JA, et al. Brazilian Portuguese validation of the university of Washington Quality of Life Questionnaire for Patients with Head and Neck Cancer. Head Neck. 2006;28(12):1115-21.

10. Lee YH, Lai YH, Yueh B, Chu PY, Chen YJ, Chen SC, et al. Validation of the University of Washington Quality of Life Chinese Version (UWQOL-C) for Head and Neck Cancer Patients in Taiwan. J Formos Med Assoc. 2017;116(4):1115-21.

11. Souza MT, Silva MD, Carvalho R. Revisão integrativa: o que é e como fazer. Einstein [serial on the internet]. 2010 [cited 2020 May 14];8(1):1026. Available from: https://www.scielo.br/pdf/eins/ v8n1/pt 1679-4508-eins-8-1-0102.pdf

12. Kanatas A, Singh P, Lowe D, Rogers SN. How will I be after my operation for oral cancer? $\mathrm{Br} \mathrm{J}$ Oral Maxillofac Surg. 2015;53:538-45.

13. Rana M, Kanatas A, Herzberg PY, Khoschdell $M$, Kokemueller $H$, Gellrich NC, et al. Prospective study of the influence of psychological and medical factors on quality of life and severity of symptoms among patients with oral squamous cell carcinoma. Br J Oral Maxillofac Surg. 2015;53(4):364-70.

14. Li X, Sun Q, Guo S. Functional assessments in patients undergoing radial forearm flap following hemiglossectomy. J Craniofac Surg. 2016;27(2):1725. 
15. Nueangkhota $P$, Liang $Y J$, Zheng GS, Su YX, Yang $W F$, Liao GQ. Reconstruction of tongue defects with the contralateral nasolabial island flap. J Oral Maxillofac Surg. 2016;74(4):851-9.

16. Li W, Zhang P, Li R, Liu Y, Kan Q. Radial free forearm flap versus pectoralis major pedicled flap for reconstruction in patients with tongue cancer: assessment of quality of life. Med Oral Patol Oral Cir Bucal. 2016;21(6):e737-e742.

17. Breeze J, Morrison A, Dawson D, Tipper J, Rehman K, Grew N, et al. Health-related quality of life after treatment for neoplasia of the major salivary glands: a pilot study. $\mathrm{Br} J$ Oral Maxillofac Surg. 2016;54(7):806-11.

18. Rylands J, Lowe D, Rogers SN. Outcomes by area of residence deprivation in a cohort of oral cancer patients: survival, health-related quality of life, and place of death. Oral 0ncol. 2016;(52):30-6.

19. Yang Y, Li F, Li W. Factors that affect the quality of life of patients with oral cancer who have had their defects reconstructed immediately after excision of the tumour. Br J Oral Maxillofac Surg. $2016 ; 54(4): 410-4$.

20. Kobayashi W, Kukobota K, Ito R, Sakaki H, Nakagawa H, Teh BG. Can superselective intraarterial chemoradiotherapy replace surgery followed by radiation for advanced cancer of the tongue and floor of the mouth? J Oral Maxillofac Surg. $2016 ; 74(6): 1248-54$.

21. Sakthivel $P$, K Irugu DV, Singh $C A$, Verma $H$, Yogal $R$, Jat $B$, et al. Quality of life outcome measures using University of Washington questionnaire version 4 in early T1/T2 anterior tongue cancers with and without radiotherapy: a cross-sectional study. Indian J Cancer. 2017;54(2):447-52.

22. Nemeth D, Zaleczna L, Huremovic A, Engelmann J, Poeschl PW, Strasz M, et al. Importance of chewing, saliva, and swallowing function in patients with advanced oral cancer undergoing preoperative chemoradiotherapy: a prospective study of quality of life. Int J Oral Maxillofac Surg. 2017;46(10):122936 .

23. Becker C, Pfeiffer J, Lange K, Dahlem KKK. Health-related quality of life in patients with major salivary gland carcinoma. Eur Arch 0to-RhinoLaryngol. 2018;275(4):997-1003.

24. Soares JRN, Dias FL, Lima RRMA, Toscano UB, Pontes ACP, Botinelly RD, et al. Assessment of quality of life in patients with advanced oral cancer who underwent mandibulectomy with or without bone reconstruction. AMB Rev Assoc Méd Bras [serial on the internet]. 2018 [cited 2020 May 14];64(8):7106. Available from: https://www.scielo.br/pdf/ramb/ v64n8/1806-9282-ramb-64-8-0710.pdf
25. Huang $T$, Liu $H$, Zhang $P$, Zhao Y. Effect of induction chemotherapy on the quality-of-life in patients with advanced stage tongue cancer by a prospective study. J Int Med Res. 2018;46(4):14616.

26. Breeze J, Rennie A, Dawson D, Tipper J, Rehman KU, Grew N, et al. Patient-reported quality of life outcomes following treatment for oral cancer. Int $\mathrm{J}$ Oral Maxillofac Surg. 2018;47(3):296-301.

27. Miranda FA, Araújo L0, Melo MR, Barbosa RC, Caldeira AP, Oliveira FPSL. Políticas públicas em saúde relacionadas ao diagnóstico precoce e rastreamento do câncer bucal no Brasil. Sanare (Sobral, Online) [serial on the internet]. 2019 [cited 2020 May 14];18(2):86-95. Available from: https://sanare. emnuvens.com.br/sanare/article/view/1378/703

28. Kalavrezos N, Scully C. Mouth cancer for clinicians part 12: cancer treatment (chemotherapy and targeted therapy). Dent Update. 2016;43(6):567-74.

29. Deng H, Sambrook PJ, Logan RM. The treatment of oral cancer: an overview for dental professionals. Aust Dent J. 2011;56(3):244-52.

30. Kamrani P, Sadiq NM. Anatomy, head and neck, oral cavity (mouth) [document on the internet]. 2019 [cited 2020 May 14]. Available from: https:// www.ncbi.nlm.nih.gov/books/NBK545271/

31. Kalavrezos N, Scully C. Mouth cancer for clinicians part 10: cancer treatment (surgery). Dent Update. $2016 ; 43(4): 375-87$.
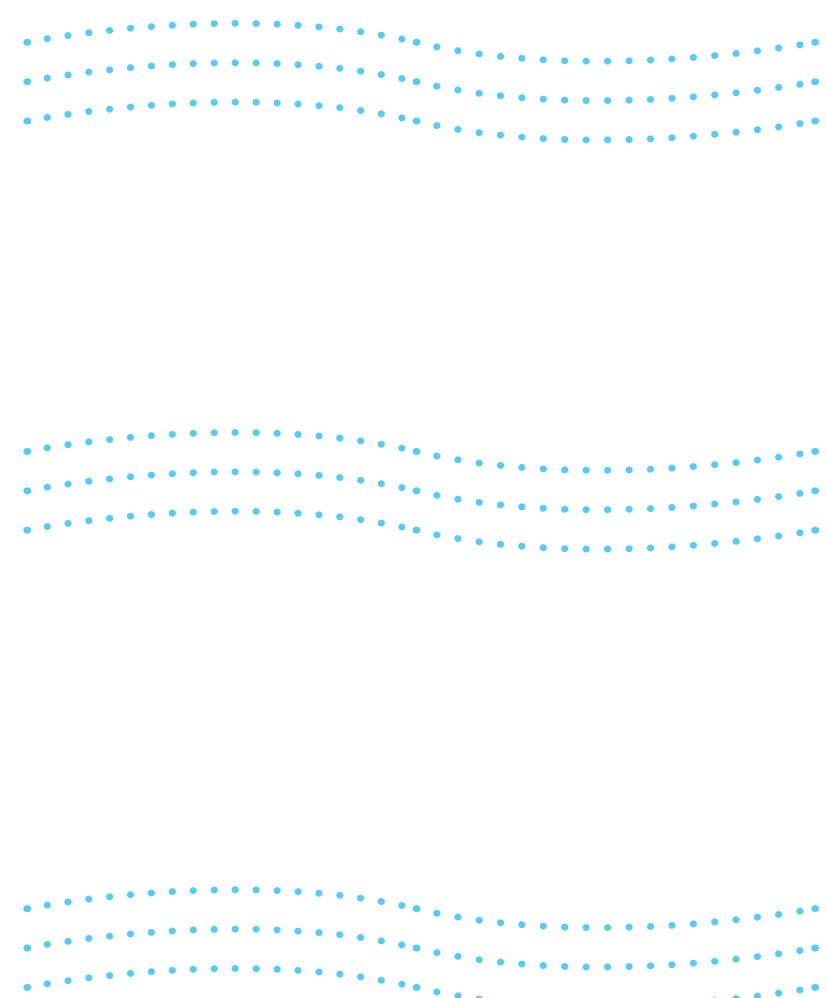